\title{
Reabilitação auditiva por aparelhos de amplificação sonora individual (AASI): perfil epidemiológico de pacientes adaptados em um hospital terciário em 5 anos
}

\section{Audiologic rehabilitation with hearing aids: epidemiological profile of fitted patients at a tertiary hospital in 5 years}

Raphael Oliveira Correia ${ }^{1}$. Caio Calixto Diógenes Pinheiro ${ }^{1}$. Felipe Cordeiro Gondim de Paiva ${ }^{1}$. Pedro Sabino Gomes Neto ${ }^{2}$. Talita Parente Rodrigues ${ }^{3}$. Alessandra Teixeira Bezerra de Mendonça ${ }^{3}$. Marcos Rabelo de Freitas ${ }^{4}$.

1 Médico residente em Otorrinolaringologia, Hospital Universitário Walter Cantídio (HUWC), Fortaleza, Ceará, Brasil. 2 Graduando em Medicina, Universidade Federal do Ceará (UFC), Fortaleza, Ceará, Brasil. 3 Fonoaudióloga, Hospital Universitário Walter Cantídio (HUWC), Fortaleza, Ceará, Brasil. 4 Doutor em cirurgia, Médico Otorrinolaringologista, Departamento de Cirurgia, Universidade Federal do Ceará (UFC), Fortaleza, Ceará, Brasil.

\section{RESUMO}

Objetivo: avaliação do perfil epidemiológico dos pacientes beneficiados com aparelhos auditivos em um hospital terciário, identificando-se seu número, idade, características da perda auditiva, tempo de espera pelo AASI, ganho funcional e tempo de seguimento no serviço. Métodos: revisão dos prontuários de pacientes que receberam sua primeira prótese auditiva entre os anos de 2006 e 2010. Resultados: foram avaliados 388 prontuários, apresentando idades entre 1 e 96 anos (média 56,7). Houve predomínio de perdas neurossensoriais $(74,4 \%)$ e de grau moderado $(59,2 \%)$. As principais etiologias foram presbiacusia $(21,6 \%)$ e otites médias crônicas (11,1\%). O tempo de seguimento variou de 1 a 4 anos e $28,1 \%$ dos pacientes beneficiados mantiveram retornos ambulatoriais regulares. Conclusão: o maior grupo de pacientes beneficiados foi de idosos com presbiacusia e perdas neurossensoriais de grau moderado. O ganho funcional foi superior a $15 \mathrm{~dB}$ NA para a maioria dos pacientes. $\mathrm{O}$ tempo de seguimento médio foi de 2,5 anos e a reposição dos aparelhos ocorreu em quase metade dos pacientes em acompanhamento regular.

Palavras-chave: Aparelho auditivo. Audiologia. Correção de deficiência auditiva.

\section{ABSTRACT}

Objective: evaluation of the epidemiological profile of patients fitted with hearing aids at a tertiary hospital, specifically their number and age, characteristics of hearing loss, waiting time for the fitting of the device, functional gain and follow up time. Methods: review of medical records of patients who were fitted with their first air conduction hearing aid from 2006 to 2010. Results: we evaluated 388 records with age range 1 to 96 years (mean 56.7). Sensorineural (74.4\%) and moderate (59.2\%) hearing loss were predominant. The main etiologies were presbycusis $(21.6 \%)$ and chronic otitis media (11.1\%). Follow-up time ranged from 1 to 4 years and $28.1 \%$ of fitted patients regularly attended follow-up service. Conclusion: the largest group of patients using hearing aids consisted of elderly patients with presbycusis and moderate sensorineural hearing loss. Functional gain was greater than $15 \mathrm{~dB}$ HL for most patients. The mean follow-up period was 2.5 years and the replacement of hearing aids occurred in almost $50 \%$ of patients who regularly attended follow-up.

Keywords: Audiology. Correction of hearing impairment. Hearing aids.

Autor correspondente: Raphael Oliveira Correia, Rua Sigefredo Pinheiro 100, ap 201, Bloco Audízio B, Fátima, Fortaleza, Ceará. CEP: $60415-160$. Telefone: +55 85 3257-4453/99905-2180. E-mail: raphaelcore@yahoo.com.br

Conflito de interesses: Não há qualquer conflito de interesses por parte de qualquer um dos autores.

Recebido em: 15 Jun 2016; Revisado em: 05 Set 2016; Aceito em: 10 Out 2016. 


\section{INTRODUÇÃO}

Segundo dados do último censo brasileiro, a deficiência auditiva (DA) é a terceira mais frequente, afetando em torno de 9,5 milhões de pessoas, o que corresponde a $5 \%$ da população. ${ }^{1}$ Levantamentos americanos apontam prevalências aproximadas de DA em $20 \%$ da população adulta. ${ }^{2}$

$\mathrm{Na}$ infância, a ausência dos estímulos sonoros prejudica o desenvolvimento de um sistema sensorial e cognitivo funcional. ${ }^{3-5}$ Enquanto em adultos pode associar-se a declínio cognitivo, depressão, baixo rendimento escolar e produtividade. ${ }^{6}$ Em ambos os casos, a detecção precoce da deficiência e a recuperação da função auditiva são fundamentais na melhora da qualidade de vida desses pacientes.

Reabilitação auditiva consiste na recuperação das qualidades e habilidades prévias a perda, sendo frequentemente realizada através de aparelhos de amplificação sonora individual (AASI). Estes atuam amplificando o som nas frequências em que a audição encontra-se comprometida com o objetivo de corrigir ou minimizar as limitações que a deficiência acarreta e reinserir o paciente em seu meio social, com mínimo de desconforto auditivo e estético para o usuário. ${ }^{7}$

Segundo as diretrizes do sistema único de saúde (SUS) para o fornecimento de AASI, podem ser beneficiados pacientes com perda auditiva permanente confirmada por audiometria. Este exame deve apresentar média dos limiares tonais nas frequências de 500, 1.000, 2.000 e $4.000 \mathrm{~Hz}$ maior que $40 \mathrm{~dB}$ NA em adultos e maior que $30 \mathrm{~dB}$ NA em menores de 15 anos, considerando a via aérea da melhor orelha. ${ }^{8}$ Existem ainda indicações relativas, avaliadas caso a caso. Estes critérios foram atualizados em 2012, passando a considerar apenas as frequências $500,1.000$ e $2.000 \mathrm{~Hz}$ no cálculo da média tonal. ${ }^{9,10}$

Os serviços de Otorrinolaringologia e Fonoaudiologia do Hospital Universitário Walter Cantídio organizaram o primeiro programa de próteses auditivas vinculado ao SUS do estado do Ceará, sendo ainda um dos pioneiros do Brasil. Iniciado em 1999, é anterior a normatização da política nacional de atenção a saúde auditiva, em $2004 .^{8}$ Tal programa é composto de fases que envolvem diagnóstico da deficiência auditiva, seleção do AASI, adaptação deste e acompanhamento periódico do paciente adaptado. ${ }^{11}$ Cerca de 2000 pacientes já foram beneficiados até o ano de 2015.

O atendimento a este grupo de pacientes possibilitou a aquisição de um grande banco de dados acerca das características das perdas auditivas e das dificuldades no processo de adaptação aos aparelhos auditivos. Tais informações são importantes para identificar fatores passíveis de prevenção da deficiência auditiva, possibilitar melhorias no atendimento e permitir o planejamento futuro dos serviços de saúde.

Este estudo tem por objetivo avaliar o perfil epidemiológico dos pacientes que receberam aparelhos auditivos em um hospital terciário entre os anos de 2006 e 2010. Busca-se a identificação do seu número; faixa etária; grau, tipo e etiologia da perda auditiva; tempo de espera pelo aparelho; ganho funcional e tempo de seguimento no serviço.

\section{MÉTODOS}

A pesquisa foi previamente aprovada pelo comitê de ética vinculado à instituição onde o levantamento de dados foi realizado (CAAE: 36762314.4.0000.5045).

Realizou-se a revisão dos prontuários de pacientes beneficiados com sua primeira prótese auditiva de transmissão aérea no período de 2006 a 2010. Foram excluídos os prontuários com informações incompletas aos principais objetivos da pesquisa e aqueles em que a indicação do aparelho auditivo não tenha se baseado em avaliação audiométrica.

Para fins de padronização das respostas foi considerado como tempo de espera pelo AASI o intervalo entre a entrevista social do paciente e a data de compra do mesmo pela instituição, por serem informações confiáveis e presentes em todos os prontuários. Estabeleceu-se como perda de seguimento a ausência de retornos ambulatoriais em dois anos consecutivos. A classificação da perda auditiva ocorreu conforme descrito nos Quadros 1 e 2. ${ }^{12,13}$ A avaliação do ganho funcional foi realizada através de duas medidas. A primeira pela diferença entre as médias dos limiares tonais nas frequências 500, 1.000, 2.000 e $4.000 \mathrm{~Hz}$ obtidos em audiometria de campo livre com e sem aparelho. A segunda pela diferença do limiar de reconhecimento de fala (LRF) obtido com e sem aparelho. Em ambas avaliaram-se as respostas da melhor orelha aparelhada.

Os prontuários foram revisados através de um questionário eletrônico e agrupados em um banco de dados (Plataforma Júpiter $\left.^{\circledR}\right)$. A análise foi realizada na Plataforma Júpiter ${ }^{\circledR}$ e no software SPSS Statistics ${ }^{\circledR} 20.0 .0$.

\section{RESULTADOS}

Dos 453 prontuários avaliados, 388 foram incluídos na pesquisa. Alguns parâmetros foram encontrados em um número menor de prontuários devido a informações incompletas nestes, o que será especificado conforme ocorrer. As mulheres (220) tiveram predomínio em relação aos homens (168), com relação 1,3:1. A faixa etária variou de 1 a 96 anos, com média de 56,7 e desvio-padrão de 24. A maioria dos pacientes procederam da capital e região metropolitana (334 ou $86,1 \%$ ), o restante do interior do estado. Os dados relativos à escolaridade e etiologia da perda auditiva estão descritos nos Gráficos 1 e 2.

Todos os pacientes possuíam perdas bilaterais. Considerando cada orelha individualmente $(\mathrm{N}=776)$, predominaram as perdas neurossensoriais $(74,4 \%)$, seguidas das mistas $(23,1 \%)$ e condutivas $(2,6 \%)$. Com relação ao grau de perda auditiva, distribuíram-se da seguinte forma: moderada $(59,3 \%)$, severa $(15,3 \%)$, leve $(14,9 \%)$, profunda $(9,2 \%)$ e anacusia $(1,3 \%)$. A maioria dos pacientes possuía otoscopia normal $(85,4 \%)$ e não havia realizado cirurgias otológicas 
prévias $(80,16 \%)$. As principais alterações à otoscopia estão discriminadas na Tabela 1 .

Os dados do estudo são referentes a um período em que a indicação do aparelho auditivo era realizada avaliando as médias dos limiares tonais da via aérea nas frequências de 500, $1.000,2.000$ e $4.000 \mathrm{~Hz}$ na melhor orelha. Os novos critérios de indicação são válidos desde 2012 e deixam de incluir a frequência de $4.000 \mathrm{~Hz}$ no cálculo da média. Se os novos critérios fossem aplicados ao grupo de pacientes da pesquisa, 91,6\% destes teriam a indicação inalterada, 7,9\%

Quadro 1. Classificação do grau de perda auditiva utilizada.

\begin{tabular}{|l|l|}
\hline Grau de perda auditiva & $\begin{array}{l}\text { Média dos limiares tonais audiométricos } \\
\text { da via aérea nas frequências 0,5; 1 e 2 } \\
\text { kHz (dB NA) }\end{array}$ \\
\hline Leve & 26 a 40 \\
\hline Moderada & 41 a 70 \\
\hline Grave & 71 a 90 \\
\hline Profunda & $>90$ \\
\hline Anacusia & $\begin{array}{l}\text { Ausência de respostas até intensidade } \\
\text { de } 120 \text { dB NA }\end{array}$ \\
\hline
\end{tabular}

Fonte: adaptado de Conselho Federal de Fonoaudiologia (CFFa), 2009.

Gráfico 1. Grau de escolaridade dos pacientes usuários de aparelhos auditivos, Hospital Universitário Walter Cantídio, 2006-2010.

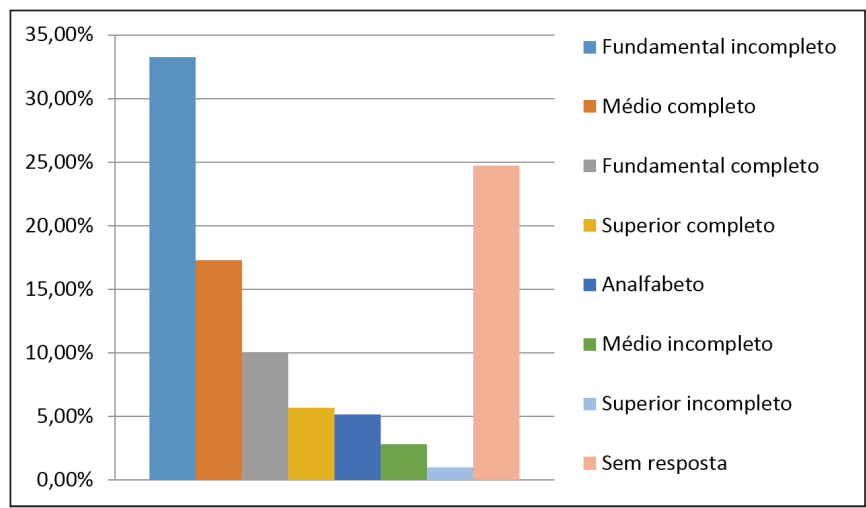

Tabela 1. Otoscopias alteradas em usuários de aparelhos auditivos, Hospital Universitário Walter Cantídio, 2006-2010.

\begin{tabular}{lcc}
\hline Alteração Otoscópica & $\mathrm{n}$ & $\%$ \\
\hline Perfuração timpânica & 45 & 5,8 \\
Retração timpânica & 17 & 2,2 \\
Cavidade otomastoidea & 15 & 1,9 \\
Atelectasia timpânica & 6 & 0,8 \\
\hline
\end{tabular}

dos pacientes deixaria de receber o AASI e $0,5 \%$ passariam a receber. Este último cálculo foi feito com 382 pacientes, nos quais as duas médias puderam ser identificadas.

Os pacientes foram beneficiados com próteses auditivas bilaterais na quase totalidade dos casos $(94,9 \%)$, sendo o tipo retroauricular o mais frequentemente fornecido $(58,3 \%)$ seguido do intracanal $(31,2 \%)$. O tempo de espera entre a avaliação audiológica e o recebimento do aparelho variou entre 1 e 28 meses, com média de 5,4 e desvio-padrão de 4,7. Os valores do ganho funcional observado estão discriminados na Tabela 2 .

Quadro 2. Classificação do tipo de perda auditiva utilizada.

\begin{tabular}{|l|l|}
\hline Tipo de perda auditiva & $\begin{array}{l}\text { Comparação dos limiares tonais } \\
\text { audiométricos nas vias aérea (VA) } \\
\text { e óssea (VO) em pelo menos duas } \\
\text { frequências consecutivas (dB NA) }\end{array}$ \\
\hline Condutiva & $\begin{array}{l}\text { VA com limiar }>25 \text { e VO preservada } \\
\text { até 15 }\end{array}$ \\
\hline Neurossensorial & $\begin{array}{l}\text { VA com limiar }>25 \text { e VO com perda } \\
\text { maior que 15, com diferença aero-óssea } \\
\text { de até 10 }\end{array}$ \\
\hline Mista & $\begin{array}{l}\text { VA com limiar }>25 \text { e VO com perda } \\
\text { maior que 15, com diferença aero- } \\
\text { óssea maior que } 10\end{array}$ \\
\hline
\end{tabular}

Fonte: adaptado de Conselho Federal de Fonoaudiologia (CFFa), 2009.

Gráfico 2. Etiologias de perda auditiva identificadas em usuários de aparelhos auditivos, Hospital Universitário Walter Cantídio, 2006-2010.

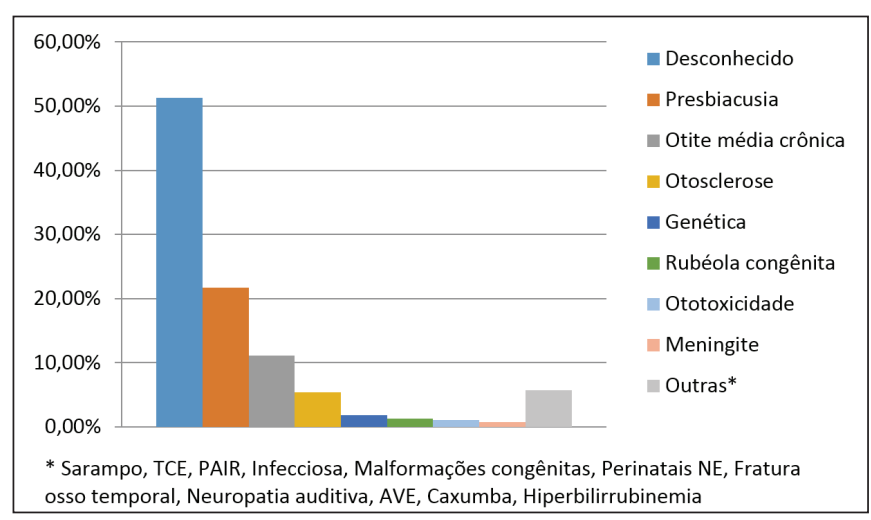

Tabela 2. Ganho funcional dos pacientes usuários de aparelhos auditivos, Hospital Universitário Walter Cantídio, 2006-2010.

\begin{tabular}{llrl}
\hline & $\begin{array}{l}\text { Diferença entre } \\
\text { o limiar } \\
\text { reconhecimento } \\
\text { fala com/sem AASI } \\
\text { de NA) }\end{array}$ & $\begin{array}{l}\text { Diferença entre as } \\
\text { médias tonais em 0.5, } \\
\text { 1 e 2 kHz em campo } \\
\text { livre, com/sem AASI } \\
\text { (dB NA) }\end{array}$ \\
\hline Pacientes avaliados & $80(17,4 \%)$ & $180(39,1 \%)$ \\
Amplitude & $5-50$ & $5-45$ \\
Q1 & 15 & 15 \\
Mediana & 20 & 20 \\
Q3 & 25 & 25 \\
\hline
\end{tabular}


Após o recebimento da prótese auditiva, 28,1\% tiveram retornos ambulatoriais em um grupo de 388 pacientes. O tempo de seguimento variou de 1 a 4 anos, com média de 2,5. A reposição do AASI ocorreu em 45 indivíduos, o que equivale a $11,6 \%$ do total do estudo e $41,3 \%$ dos que mantiveram o seguimento no serviço.

\section{DISCUSSÃO}

Houve predominância de pacientes aparelhados idosos, com perdas auditivas neurossensoriais pós-linguais de grau moderado, com pequena predominância de mulheres, em concordância com outros levantamentos semelhantes realizados. ${ }^{14,15}$

A etiologia principal identificada, presbiacusia, reflete a faixa etária principal. ${ }^{15}$ Diante da tendência atual de envelhecimento da população brasileira espera-se um aumento na demanda dos serviços de saúde nos próximos anos com relação ao atendimento dessa faixa etária e de suas comorbidades características, sendo a presbiacusia a terceira mais comum alteração fisiológica diagnosticada em idosos. ${ }^{16}$

Ainda com relação a etiologia, chama atenção a quantidade de hipoacusias secundárias a otites crônicas identificadas, figurando como segunda maior prevalência neste estudo. Uma possível explicação para este achado pode ser a de que o programa de aparelhos auditivos está inserido dentro de um serviço de referência em otorrinolaringologia para o estado, no qual são recebidos e tratados muitos casos de doenças otológicas crônicas, como as otites colesteatomatosas. Após avaliação e tratamento da doença de base, muitos destes indivíduos são encaminhados para a protetização auditiva para otimização da audição residual.

Todos os pacientes incluídos no estudo possuíam perda auditiva bilateral por ser este um critério para recebimento do aparelho auditivo. No entanto, pouco menos que $6 \%$ dos indivíduos receberam AASI unilateral. Provavelmente tais casos representam anacusia ou perda profunda de longa data em uma das orelhas, condições em que o benefício da prótese auditiva seria limitado.

\section{REFERÊNCIAS}

1. Brasil. Ministério do Planejamento, Orçamento e Gestão. Instituto Brasileiro de Geografia e Estatística - IBGE. Censo demográfico 2010: características gerais da população, religião e pessoas com deficiência. Rio de Janeiro: IBGE; 2012.

2. Hearing Loss Association of America. Basic facts about hearing loss. [Internet]. Washington (DC): HLLA; ${ }^{\circledR} 2005-2017$ [acesso em: 25 maio 2014]. Disponível em: http://www.hearingloss.org/content/ basic-facts-about-hearing-loss

3. Novaes BC, Mendes BC. Habilitação auditiva: intervenção em bebês e crianças pequenas. In: Caldas S Neto, Mello JF Júnior, Martins RH, Costa SS, organizadores. Tratado de otorrinolaringologia e cirurgia cervicofacial. 2. ed. v. 2, Otologia - Otoneurologia. São Paulo: Roca; 2011. p. 371-80.

4. Chermak GD, Bellis JB, Musiek FE. Neurobiology, cognitive
A atualização dos critérios de indicação do aparelho auditivo no SUS, passando a avaliar somente as frequências da fala, parece levar a uma redução no número de indivíduos beneficiados, que no caso foi de 7,9\%. As perdas auditivas tendem a envolver mais precocemente as frequências agudas, como ocorre na presbiacusia, ${ }^{17}$ principal etiologia do estudo, o que poderá determinar um retardo na reabilitação auditiva destes indivíduos.

A maior quantidade de aparelhos retroauriculares encontrada está em consistência com o predomínio de idosos no grupo, ${ }^{18}$ já que este tipo de AASI é o de mais fácil manipulação para estes pacientes além de ser o preferido nas perdas moderadas a graves. O ganho funcional apresentou valores aceitáveis para esta forma de reabilitação, sendo superior a 20 dB NA em quase metade dos pacientes.

A taxa de perda de seguimento encontrada no programa, em torno de $70 \%$, mostrou-se superior à relatada na literatura, de 40 a $57 \% .{ }^{19-21}$ Os possíveis fatores contribuintes para este dado são a maior taxa de idosos observada, baixa escolaridade e baixo nível socioeconômico, fatores adversos no processo de adaptação ao AASI. ${ }^{20}$

\section{CONCLUSÃO}

Os aparelhos auditivos de condução aérea são uma das principais formas de reabilitação auditiva, especialmente no âmbito do serviço público. O maior grupo de pacientes beneficiados identificados neste estudo foi de idosos com presbiacusia e perdas neurossensoriais de grau moderado. A segunda etiologia observada foram as perdas secundárias a otites médias crônicas.

A aplicação dos novos critérios de indicação de AASI ao grupo da pesquisa reduziria o número de indivíduos beneficiados. O ganho funcional foi superior a $15 \mathrm{~dB}$ NA para a maioria dos pacientes. Dos pacientes que recebem a prótese auditiva, pouco mais de um quarto retorna ao serviço para o acompanhamento periódico. O tempo de seguimento médio foi de 2,5 anos e a reposição dos aparelhos ocorreu em quase metade dos pacientes em acompanhamento regular.

science and intervention. In: Chermak GD, Musiek FE, editores. Handbook of central auditory processing disorder: comprehensive intervention. 1. ed. San Diego: Plural; 2007. p. 3-28.

5. Vouloumanos A, Werker JF. Listening to language at birth: evidence for a bias for speech in neonates. Dev Sci. 2007;10(2):159-64.

6. Cacciatore F, Napoli C, Abete P, Marciano E, Triassi M, Rengo F. Quality of life determinants and hearing function in an elderly population: Osservatorio Geriatrico Campano Study Group. Gerontology. 1999;45(6):323-8.

7. Piza MR. Aparelho de amplificação sonora individual: aspectos de ordem prática para o otorrinolaringologista. In: Caldas S Neto, Mello JF Júnior, Martins RH, Costa SS, organizadores. Tratado de otorrinolaringologia e cirurgia cervicofacial. 2. ed. v.2, Otologia Otoneurologia. São Paulo: Roca; 2011. p. 346-57. 
8. Brasil. Ministério da Saúde. Secretaria de Atenção à Saúde. Portaria $\mathrm{n}^{\circ} .587$, de 07 de outubro de 2004. Dispõe sobre a organização e a implantação das redes estaduais de atenção à saúde auditiva. Diário Oficial da União. 11 out 2004; Seção 1 n(196):pag 105.

9. Brasil. Ministério da Saúde. Portaria $n^{0} 793$, de 24 de abril de 2012. Institui a rede de cuidados à pessoa com deficiência no âmbito do sistema único de saúde. Diário Oficial da União. 25 abr 2012; Seção 1 n(80):pag 94.

10. Brasil. Ministério da Saúde. Portaria $n^{\circ} 835$, de 25 de abril de 2012. Institui incentivos financeiros de investimento e de custeio para o componente atenção especializada da rede de cuidados à pessoa com deficiência no âmbito do sistema único de saúde. Diário Oficial da União. 26 abr 2012; Seção 1 n(81):pag 50.

11. Pinheiro SD. Tratamento das deficiências auditivas. In: Pinheiro $\mathrm{SD}$, Freitas MR, Nunes AA, editores. Otorrinolaringologia para a graduação. 2. ed. Fortaleza: UFC; 2006. p. 241-51.

12. Griz SM, Menezes DC, Menezes PL. Audiometria tonal e logoaudiometria. In: Caldas S Neto, Mello JF Júnior, Martins RH, Costa SS, organizadores. Tratado de otorrinolaringologia e cirurgia cervicofacial. 2. ed. v. 1, Otologia - Otoneurologia. São Paulo: Roca; 2011. p. 389-411.

13. Conselho Federal de Fonoaudiologia (CFFa). Audiometria tonal, logoaudiometria e medidas de imitância acústica: orientações dos Conselhos de Fonoaudiologia para o laudo audiológico. Brasília: CFFa; 2009.

14. Gresele AD, Lessa AH, Alves LC, Torres EM, Vaucher AV, Moraes
$\mathrm{AB}$, et al. Levantamento e análise de dados de pacientes atendidos em um programa de concessão de aparelhos de amplificação sonora individual. CoDAS. 2013;25(3):195-201.

15. Santos MA, Vianna MF, Marotta L, Almeida CM, Sousa OM Neto. A deficiência auditiva em hospital terciário: estudo epidemiológico. Braz J Otorhinolaryngol. 2008;74(1):222-6.

16. Jardim IS, Iwahashi JH, Paula VC. Study of the audiological profile of individuals attended in a brazilian diagnostic service. Int Arch Otorhinolaryngol. 2010;14(1):32-7.

17. Gândara ME, Alves FR. Presbiacusia. In: Caldas S Neto, Mello JF Júnior, Martins RH, Costa SS, organizadores. Tratado de otorrinolaringologia e cirurgia cervicofacial. 2. ed. São Paulo: Roca; 2011. p. 302-13.

18. Mondelli MF, Silva LS. Profile of the patients serviced in a high complexity system. Int Arch Otorhinolaryngol. 2011;15(1):29-34.

19. Knudsen LV, Oberg M, Nielsen C, Naylor G, Kramer SE. Factors influencing help seeking, hearing aid uptake, hearing aid use and satisfaction with hearing aids: a review of the literature. Trends Amplif. 2010;14(3):127-54.

20. Lupsakko TA, Kautiainen HJ, Sulkava R. The non-use of hearing aids in people aged 75 years and over in the city of Kuopio in Finland. Eur Arch Otorhinolaryngol. 2005;262(3):165-9.

21. Gianopoulos I, Stephens D, Davis A. Follow-up of people fitted with hearing aids after adult hearing screening: the need for support after fitting. BMJ. 2002;325(7362):471.

\section{Como citar:}

Correia RO, Pinheiro CC, Paiva FC, Gomes PS Neto, Rodrigues TP, Mendonça AT, et al. Reabilitação auditiva por aparelhos de amplificação sonora individual (AASI): perfil epidemiológico de pacientes adaptados em um hospital terciário em 5 anos. Rev Med UFC. 2017 mai-ago;57(2):26-30. 